CASE REPORT

\title{
Oral phaeohyphomycosis
}

\section{S V Cardoso, S S Campolina, A L S Guimarães, P R Faria, E M da C Costa, R S Gomez, A Rocha, R B Caligiorne, A M Loyola}

J Clin Pathol 2007;60:204-205. doi: 10.1136/jcp.2005.035808

This is the first description of solitary phaeohyphomycosis affecting the mucosal surface. The lesion developed in the inferior lip of a 57-year-old woman. After surgical resection, histopathological examination evidenced characteristic brownish fungal structures within granulomatous-purulent inflammation. Amplification and sequencing of rDNA obtained from paraffin-embedded tissue identified Alternaria species, as the causative agent.

$P$ haeohyphomycosis is a dematiaceous fungal infection. ${ }^{12}$ Bipolaris, Curvularia, Exserohilum and Alternaria species are often involved in human infections. ${ }^{13}$ These are primarily soil saprophytes, plant pathogens and domestic environment contaminants. Skin lesions of traumatic origin affecting the hands and feet are the most common presentation in healthy people. $^{12}$ Systemic phaeohyphomycosis is almost restricted to immunocompromised individuals, with fatal outcome common. ${ }^{4}$ Solitary lesions can be treated by surgical excision, and disseminated disease requires systemic medical treatment. $^{14-6}$

The histopathology is usually diagnostic, and microbiological culture is required to guide treatment. Polymerase chain reaction (PCR) assays and analysis of the internal transcribed spacer of ribosomal DNA represent a powerful tool for rapid and precise laboratory diagnosis of invasive fungal infections. ${ }^{78}$

This paper represents the first description of phaeohyphomycosis affecting oral mucosa. The lesion had an ordinary presentation shared by common oral lesions. Histopathology was diagnostic, and recently developed molecular protocols were used to determine the causative agent.

\section{CASE REPORT}

\section{The patient}

A 57-year-old woman presented to the Dermatology Division of the Federal University of Uberlândia (Uberlândia, Brazil) for an asymptomatic swelling on the inner surface of the right side of her inferior lip. It was first noticed 2 weeks before the first appointment, and the lesion had grown until slight deformity compelled the patient to seek professional care. When specifically asked, she denied direct trauma to the mucosa. However, the patient chronologically associated the lesion with the use of a herbal infusion as a self-medication against renal infection. Besides drinking the beverage, the patient also chewed the infused foliage. According to her, the renal condition was efficiently treated with levofloxacin $(500 \mathrm{mg} /$ day) before the appointment at our institution. She was otherwise healthy except for the persistence of the oral lesion. Further, her medical history was not contributory. On clinical examination, a deep and painless $0.5-\mathrm{cm}$ nodule was observed. It was firm and well delineated on palpation. Cutaneous and mucosal surfaces were normal. No other oral or systemic changes were seen. The clinical presentation led to the hypothesis of a pleomorphic adenoma, and surgical excision was proposed as the therapeutic approach. It was conducted without delay, and a well-delineated, dense nodule of whiteyellowish colour was obtained.

\section{Pathology}

Sections stained with haematoxylin-eosin (fig 1) disclosed focal granulomatous-purulent inflammation, depicting histiocytic cells, a few multinucleated giant cells and lymphocytes, intermingled with polymorphonuclear neutrophils and liquefactive necrosis. Surprisingly, thick-walled, septate hyphae and yeast-like structures were also observed, some of them with brown pigmentation, either inside areas of abscess formation or within a salivary gland ectatic duct, denoting a dematiaceous fungal infection. These mycotic structures were also seen in periodic acid-Schiff and Groccott-Gomori methenamine silver stains, confirming the diagnosis of phaeohyphomycosis. Once the lesion was entirely removed, it was not possible to perform culture for species confirmation.

The patient was then referred to the Infectious Diseases Division, but no treatment was necessary, as there were no other clinical changes. After 2 years follow-up, the patient remained completely free of the disease.

\section{Molecular diagnosis}

As biopsy for microbiological culture was not performed, and the patient could not retrieve the vegetable she used, DNA was extracted from the paraffin preparation. ${ }^{9}$ Briefly, 10 sections of $10 \mu \mathrm{m}$ thickness were obtained. After transfer to microtubes, the sample was bathed thrice in xylene at $65^{\circ} \mathrm{C}$ for $10 \mathrm{~min}$. The material was then immersed in two baths of $95 \%$ ethanol, followed by one $70 \%$ ethanol bath. Centrifugation at $8.7 \times 10^{3} \mathrm{~g}$ for $2 \mathrm{~min}$ succeeded each bath. DNA was finally isolated with the DNeasy Tissue Kit (Qiagen, Valencia, California, USA). Internal transcribed spacer domains of rDNA were amplified with the universal primers ITS I (5'-TCCGTAGGTGAACCTGCGG-3') and ITS4 (5'-TCCTCCGCTTATTGATATGC-3'), as described previously. ${ }^{10}$ After amplification, the PCR product was purified with the QIAquick PCR Purification Kit (Qiagen) and sequenced by the Dyenamic ET Dye Terminator Kit (MegaBACE/Amersham Biosciences, Sunnyvale, California,USA). The nucleotide sequence obtained was submitted to and compared with the EMBL GenBank database (accession number: bankit754353) using the Fasta 2.0 program. High identity with about 100 sequences of the Alternaria genus was found.

\section{DISCUSSION}

Based on a literature review, this is the first report of phaeohyphomycosis in oral tissues. Further, no other case of solitary lesions affecting other mucosal surfaces was seen. Previous descriptions of the disease at other body sites have also noted solitary, asymptomatic, and well-defined plaques or

Abbreviations: $P C R$, polymerase chain reaction 

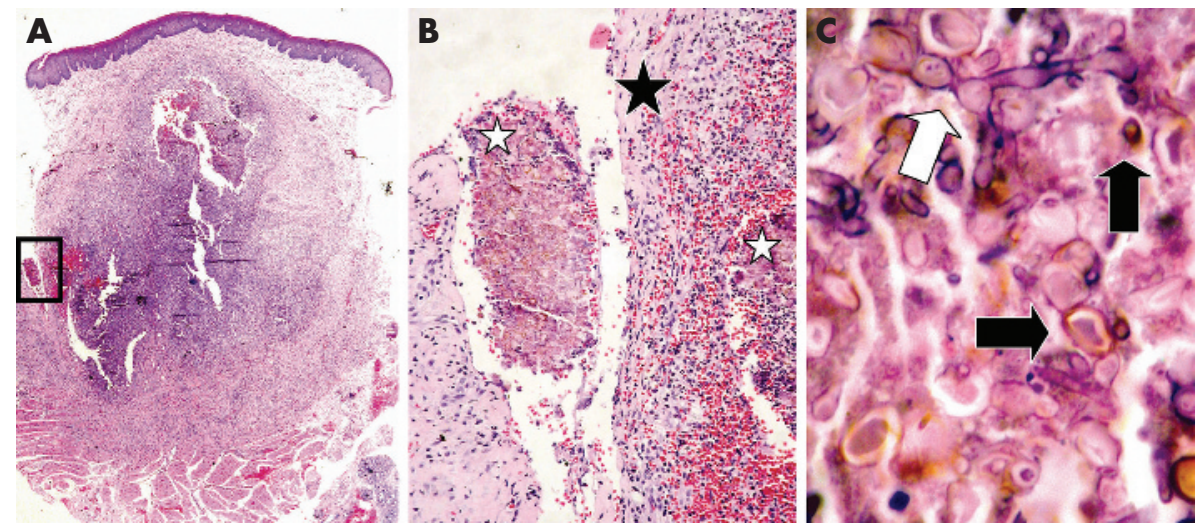

Figure 1 (A) Inflammatory reaction and microabscess and cavity formation (the so-called "phaeohyphomycotic cyst") between the oral epithelium and intrinsic muscles of the lip. The demarcated area is detailed in (B), which shows the inflamed fibrous tissue or capsule (black star) that separates two cavities containing the brown-pigmented microorganisms (white stars). No epithelial lining was seen in such cavities. (C) Thick-walled hyphae and yeast-like structures, some of them brown-pigmented (black arrows), characteristic of a phaeohyphomycotic infection. Typical narrowing close to the septa is highlighted (white arrow). Staining was done with haematoxylin and eosin. Original magnification: $A, \times 2 ; B, \times 20 ; C, \times 100$.

nodulations. ${ }^{12}$ Such clinical presentations are quite common, and may lead to incorrect diagnosis if the lesion is located in an area with a high incidence of entities such as salivary gland or mesenchymal lesions. Clinical misdiagnosis in fact occurred in the present case. In such instances, histopathology is diagnostic. Another important issue is that our patient did not recall a conclusive history of trauma to the mucosa or skin. The use of a vegetable infusion was the only suspected source of infection. Anyway, there are other reports with a negative history of trauma. $^{125}$ In our patient, it was not possible to entirely exclude that the lesion had been derived from the skin, despite the pronounced intraoral aspect. However, the identification of microorganisms and inflammation between the oral epithelium and lip musculature (fig 1) corroborates an infection through the mucosal surface. Finally, superficial presentations of the disease have been considered to be not aggressive. ${ }^{12}$

As biopsy is an important diagnostic procedure for phaeohyphomycosis, pathologists should bear in mind the unusual, but characteristic, histological presentation of the infection. The diagnosis is primarily based on small foci of brownish hyphae and yeast structures superimposed on a suppurative and granulomatous inflammatory reaction. Fungal structures are usually located near the fibrous capsule or within cavitations. ${ }^{125}$ Such a description is well applied to the present report.

The literature states that culture is required to identify the causative species. ${ }^{1}$ However, as in many other studies, we did not perform this procedure because the lesion was entirely excised and further processed, and no recurrences were noted. ${ }^{2}$ In this sense, molecular tools were used to identify Alternaria species as the aetiological agent. We believe that it may be helpful for those interested in the therapeutic management of patients with disseminated disease.

In conclusion, phaeohyphomycosis is a rare disease that can affect oral tissues, where it can mimic other lesions. Observation of brown-pigmented fungi within a granulomatous-purulent inflammatory reaction is diagnostic. The lesion is not usually invasive, and either surgery or antifungals are successful treatments.

\section{ACKNOWLEDGEMENTS}

We thank Professor Josep Guarro (Rovira I Virgili University, Spain) for his kind attention in discussing the present case.

\section{Authors' affiliations}

S V Cardoso, Oral Diagnosis Unit, State University of Montes Claros, Montes Claros, Brazil

S S Campolina, R B Caligiorne, Clinical Researches Laboratory, René Rachou Research Centre / FIOCRUZ, and Santa Casa de Misericórdia de Belo Horizonte Hospital, Minas Gerais, Brazil

A L S Guimarães, R S Gomez, Oral Pathology Department, Federal University of Minas Gerais, Minas Gerais, Brazil

S V Cardoso, P R Faria, E M da C Costa, A M Loyola, Oral Pathology Unit, Federal University of Uberlândia, Uberlândia, Brazil

A Rocha, Surgical Pathology Department, Federal University of Uberlândia, Uberlandia, Brazil

Funding: The authors received government grants from $\mathrm{CNPq}$ and FAPEMIG, Brazil.

Competing interests: None.

Patient consent was obtained for this case report to be published.

Correspondence to: Professor A M Loyola, Laboratório de Patologia Bucal, Universidade Federal de Uberlândia, Av Pará, 1720, Bloco 2U, CEP 38401-036, Uberlândia, MG/Brazil; adrianol@hc.ufu.br

Accepted 2 February 2006

\section{REFERENCES}

1 Ackerman AB, Chongchitnant N, Sanchez J, et al. eds. Phaeohyphomycosis. In: Histologic diagnosis of inflammatory skin diseases. Baltimore, MD: Williams and Wilkins Company, 1997:614-15.

2 Ziefer A, Conner DH. Phaeohyphomycotic cyst: a clinicopathologic study of twenty-five patients. Am J Trop Med Hyg 1980;29:901-11.

3 de Hoog GS. Evolution of black yeasts: possible adaptation to the human host. Antonie Van Leeuwenhoek 1993;63:105-9.

4 Revankar SG, Patterson JE, Sutton DA, et al. Disseminated phaeohyphomycosis: review of an emerging disease. Clin Infect Dis 2000;34:467-76.

5 Bogle M, Rabkin MS, Joseph AK. Mohs micrographic surgery for the eradication of phaeohyphomycosis of the hand. Dermatol Surg 2004;30:231-3.

6 Caligiorne RB, Resende MA, Melillo PH, et al. In vitro susceptibility of chromoblastomycosis and phaeohyphomycosis agents to antifungal drugs. Med Mycol 1999;37:405-9.

7 Abliz P, Fukushima K, Takizawa K, et al. Rapid identification of genus Fonsecaea by PCR with specific oligonucleotide primers. J Clin Microbiol 2003;41:873-6.

8 Caligiorne RB, Licinio P, Dupont J, et al. ITS rDNA-based phylogenetic reconstruction in black yeasts and their relatives using algorithms with and without sequence alignment. J Clin Microbiol 2005;43:2816-23.

9 Coombs NJ, Gough AC, Primrose JN. Optimization of DNA and RNA extraction from archival formalin-fixed tissue. Nucleic Acids Res 1999;27:e12.

10 White TJ, Bruns TD, Lee SB, et al. Amplification and direct sequencing of fungal ribosomal RNA genes for phylogenetics. In: Innis NA, Gelfand J, Sninsky J, et al, eds. PCR protocols: a guide to methods and applications. San Diego, CA: Academic Press, 1989:315-22. 